\title{
Influence of Hyperlipidemia on the Treatment of Supraspinatus Tendinopathy With or Without Tear
}

\author{
Jae-Min Kim, MD, Min-Wook Kim, MD, PhD, Hyun-Jung Do, MD
}

Department of Rehabilitation, Incheon St. Mary's Hospital, College of Medicine, The Catholic University of Korea, Incheon, Korea

Objective To investigate the influence of hyperlipidemia on the treatment of supraspinatus tendinopathy, with or without tear.

Methods We retrospectively reviewed the data of patients with shoulder pain and patients with supraspinatus tendinopathy, with or without tear, were included in the study. Exclusion criteria were prior shoulder surgery, prior steroid injection, neurological diseases that could lead to shoulder pain, and use of lipid-lowering medication. According to the serum lipid profiles, patients were assigned to either the hyperlipidemia or nonhyperlipidemia group. By analyzing the numeric rating scale (NRS) before treatment, and at 2 weeks and 8 weeks after treatment, we compared the difference in treatment effect between the two groups.

Results No significant baseline difference was found among the two groups for age, gender, body mass index, duration of pain, side of pain, range of motion of affected shoulder, or physical examination. On the repeatedmeasures analysis of variance, NRS scores significantly decreased with time for both groups $(\mathrm{p}<0.001)$. When analyzing the effect of time between the subjects factor, there was significant difference in the treatment effect between the two groups $(\mathrm{p}<0.001)$, namely NRS was less decreased in the hyperlipidemia group.

Conclusion We found that hyperlipidemia may be an adversely affecting factor in the treatment of supraspinatus tendinopathy with or without tear.

Keywords Rotator cuff, Hyperlipidemia, Tendinopathy

\section{INTRODUCTION}

Rotator cuff tendinopathy is a major cause of shoulder pain, and its prevalence increases with age, affecting more than $50 \%$ of the general population by the age of 60 years [1]. The pathogenesis of rotator cuff lesions is currently under debate; however, several intrinsic and extrinsic factors are believed to play important roles in the

Received January 23, 2015; Accepted October 5, 2015

Corresponding author: Hyun-Jung Do

Department of Rehabilitation Medicine, Incheon St. Mary's Hospital, College of Medicine, The Catholic University of Korea, 56 Dongsu-ro, Bupyeonggu, Incheon 21431, Korea. Tel: +82-32-280-5981, Fax: +82-32-280-5992, E-mail: jung024@hanmail.net

ORCID: Jae-Min Kim (http://orcid.org/0000-0003-4217-3228); Min-Wook Kim (http://orcid.org/0000-0003-4505-809X); Hyun-Jung Do (http://orcid. org/0000-0001-7972-2548).

(c) This is an open-access article distributed under the terms of the Creative Commons Attribution Non-Commercial License (http://creativecommons.org/ licenses/by-nc/4.0) which permits unrestricted noncommercial use, distribution, and reproduction in any medium, provided the original work is properly cited. Copyright $\odot 2016$ by Korean Academy of Rehabilitation Medicine 
development of rotator cuff diseases [2]. Extrinsic factors include acromial shape, shoulder impingement, anterior glenohumeral dislocation, mechanical overuse, and several demographic factors such as age, obesity, and oral corticosteroid use [2-4]. Intrinsic factors refer to pathologic changes that lie within the rotator cuff muscle itself, including tendon degeneration, repetitive microtrauma, and hypovascularity [2]. Among the extrinsic factors, several factors including age, diabetes mellitus, obesity, and smoking, are believed to adversely affect rotator cuff healing [5].

Recently, several studies suggested that one of the extrinsic factors, hyperlipidemia, may be associated with rotator cuff disease. Abboud and Kim [6] first identified a relationship between rotator cuff disease and hypercholesterolemia. They prospectively collected serum lipid profiles on two age-matched populations: an experimental group, in which patients had ruptures of rotator cuff tendons, and a control group, in which patients had nontendon-related shoulder complaints. The reported outcome was that patients with rotator cuff tears were more likely to have hypercholesterolemia when compared with patients in the control group.

Subsequent studies performed by the same researchers suggest that hypercholesterolemia adversely affects rotator cuff tendon healing in animal models. One study suggested that hypercholesterolemia might have a detrimental biochemical effect on tendon healing in rat rotator cuff injury [7]. Another study demonstrated that hypercholesterolemia increased supraspinatus tendon stiffness and elastic modulus across multiple species [8]. However, these studies were in animal models, and did not identify the effect of hyperlipidemia on the treatment of rotator cuff disease. To our best knowledge, there is no clinical study that has investigated the influence of hyperlipidemia on rotator cuff healing or treatment.

The aim of this study was to investigate the influence of hyperlipidemia on treatment of supraspinatus tendinopathy with or without tear, since the supraspinatus tendon is the most commonly injured among rotator cuff tendons.

\section{MATERIALS AND METHODS}

We reviewed the data of patients who had visited our department for shoulder pain, between May 2013 and
November 2014. The study was conducted with approval from Incheon St. Mary's Hospital Institutional Review Board (IRB No. OC14RISI0062). The inclusion criterion was a diagnosis of supraspinatus tendinopathy with or without tear, by clinical evidence such as history and physical examination, and ultrasonography. Ultrasonographic diagnosis of supraspinatus tendinopathy was made when the thickness of the supraspinatus tendon (measured $1 \mathrm{~cm}$ proximal to the insertion of the distal supraspinatus) was greater than $8 \mathrm{~mm}$ (referring to a previous report that defined normal thickness as $6 \mathrm{~mm}$ with a standard deviation of $1.1 \mathrm{~mm}$ measured at that location) [9]. The Neer test, Hawkins-Kennedy test, Empty Can test, and passive ranges of motion (PROMs) in flexion, abduction, internal rotation, and external rotation were included in the physical examination. Exclusion criteria were prior shoulder surgery, prior steroid injection within 3 months, capsular pattern (such as frozen shoulder without rotator cuff tendinopathy), neurological diseases that could lead to shoulder pain (such as stroke, cervical radiculopathy, etc.), and the use of lipid-lowering medications.

We reviewed serum lipid profiles obtained before treatment. On the basis of lipid levels, patients were assigned to one of two groups: hyperlipidemia or non-hyperlipidemia. Patients were included in the hyperlipidemia group if they met one or more of the following criteria from the American Association of Clinical Endocrinologists' Guidelines for management of dyslipidemia and prevention of atherosclerosis [10]: total cholesterol $\geq 240 \mathrm{mg} / \mathrm{dL}$; high density lipoprotein (HDL) cholesterol $<40 \mathrm{mg} / \mathrm{dL}$ in men or $50 \mathrm{mg} / \mathrm{dL}$ in women; low density lipoprotein (LDL) cholesterol $>160 \mathrm{mg} / \mathrm{dL}$; triglyceride $>200 \mathrm{mg} / \mathrm{dL}$.

For almost all patients, primary treatment was by noninvasive management, such as activity modification, medication (including nonsteroidal anti-inflammatory drugs), exercise, and/or physical therapy. If no improvement occurred with noninvasive therapy, subacromial and/or intra-articular corticosteroid injections with ultrasonographic guidance were usually applied. A physiatrist, experienced in musculoskeletal ultrasonography, performed all the evaluations and management of shoulder pain.

We analyzed shoulder pain at the patient's first visit, and at 2 and 8 weeks after treatment, using an 11-point numeric rating scale (NRS), since NRS was the primary 
assessment scale of pain in our clinic, and a previous review identified that NRS had higher compliance rates, better responsiveness, ease of use, and good applicability relative to other pain assessment scales [11]. The changes in PROMs were evaluated. We then compared the difference on the effects of treatment, between the two groups. We also analyzed the following variables: age, gender, body mass index, presence of diabetes mellitus, duration of pain, side of pain, range of motion of the affected shoulder, impingement signs on physical examination, and concomitant rotator cuff tear on ultrasonography.

Data were analyzed statistically using the software application SPSS ver. 18.0 (SPSS Inc., Chicago, IL, USA). We compared NRS scores between the two groups at each time point using the independent t-test. Also, by analyzing the NRS scores, the treatment effects (over time) within each group and differences in the treatment effects between the two groups were examined, using repeatedmeasure analysis of variance (ANOVA); post-hoc tests were done using Bonferroni correction. Furthermore, we compared improvement in PROMs between the hyperlipidemia and non-hyperlipidemia groups using the independent-t-test. We also compared between-group baseline characteristics using the chi-square test and independent-samples t-test. Statistical significance was defined as a $\mathrm{p}$-value $<0.05$.

\section{RESULTS}

\section{Patients}

A total of 432 patients visited our department for shoulder pain during the study period, and 134 were diagnosed with supraspinatus tendinopathy. Among them, 14 patients were excluded from our criteria. Also, 21 patients were excluded because they did not come for followup visits or complete laboratory studies. Ultimately, 99 patients were included in our study; 50 were included in the non-hyperlipidemia group and the remaining 49 patients were in the hyperlipidemia group. No significant baseline difference was found between the two groups in regard to age, gender, body mass index, duration of pain, side of pain, range of motion of the affected shoulder, or impingement signs on physical examination (Table 1). However, the prevalence of diabetes mellitus in the hyperlipidemia group $(28.9 \%)$ was higher than in the nonhyperlipidemia group $(6.38 \%)$, and the difference was
Table 1. Baseline characteristics of patient population

\begin{tabular}{|c|c|c|c|}
\hline & $\begin{array}{c}\text { Non-hyper- } \\
\text { lipidemia } \\
\text { group } \\
(\mathbf{n}=\mathbf{5 0})\end{array}$ & $\begin{array}{c}\text { Hyper- } \\
\text { lipidemia } \\
\text { group } \\
(n=49)\end{array}$ & p-value \\
\hline Age (yr) & $55.6 \pm 10.3$ & $58.1 \pm 9.5$ & 0.21 \\
\hline Gender & & & 0.89 \\
\hline Male & 19 & 18 & \\
\hline Female & 31 & 31 & \\
\hline $\mathrm{BMI}\left(\mathrm{kg} / \mathrm{m}^{2}\right)$ & $23.5 \pm 2.8$ & $24.6 \pm 3.9$ & 0.16 \\
\hline $\mathrm{DM}$ & & & $0.01^{*}$ \\
\hline None & 47 & 38 & \\
\hline Present & 3 & 11 & \\
\hline $\begin{array}{l}\text { Symptom } \\
\text { duration (mo) }\end{array}$ & $2.8 \pm 2.4$ & $3.1 \pm 2.7$ & 0.55 \\
\hline Side of pain & & & 0.76 \\
\hline Right & 23 & 24 & \\
\hline Left & 27 & 25 & \\
\hline \multicolumn{4}{|l|}{ Range of motion $\left(^{\circ}\right)$} \\
\hline Flexion & $158.5 \pm 18.5$ & $151.0 \pm 27.6$ & 0.10 \\
\hline Abduction & $159.6 \pm 22.4$ & $148.7 \pm 34.4$ & 0.06 \\
\hline External rotation & $65.4 \pm 19.0$ & $58.6 \pm 20.4$ & 0.08 \\
\hline Impingement sign & & & 0.98 \\
\hline Negative & 3 & 3 & \\
\hline Positive & 47 & 46 & \\
\hline
\end{tabular}

Values are presented as mean \pm standard deviation or number.

BMI, body mass index; DM, diabetes mellitus. ${ }^{*} \mathrm{p}<0.05$.

statistically significant $(\mathrm{p}=0.01)$.

\section{Concomitant supraspinatus tear}

Of the 50 patients, 29 (58\%) patients in the non-hyperlipidemia group, and 37 of $49(75.5 \%)$ patients in the hyperlipidemia group, had supraspinatus tears on ultrasonography. We found that rotator cuff tears were more frequent in the hyperlipidemia group although statistical analysis showed no significant difference $(p=0.06)$.

\section{Pain}

Table 2 shows pain level measured by the NRS at baseline, and at 2 and 8 weeks after treatment. The NRSs of baseline and 2 weeks after treatment did not demonstrate a significant difference between the two groups ( $\mathrm{p}=0.90$ and $\mathrm{p}=0.11$, respectively). However, at 8 weeks 
Table 2. Numeric rating scale (NRS) pain scores at three time points

\begin{tabular}{lcccc}
\hline & Baseline & 2 weeks later & 8 weeks later & p-value \\
\hline Non-hyperlipidemia & $6.04 \pm 1.68$ & $3.60 \pm 1.87$ & $2.26 \pm 1.64$ & $<0.001^{* * * \mathrm{~b})}$ \\
& & & & $<0.001^{* * * \mathrm{c})}$ \\
Hyperlipidemia & $6.00 \pm 1.63$ & $4.26 \pm 2.22$ & $3.63 \pm 1.97$ & $<0.001^{* * * \mathrm{~b})}$ \\
p-value & $0.90^{\mathrm{a})}$ & $0.11^{\mathrm{a})}$ & $\left.<0.001^{* * *} \mathrm{a}\right)$ & \\
\hline
\end{tabular}

Values are presented as mean \pm standard deviation.

${ }^{*} \mathrm{p}<0.05,{ }^{* *} \mathrm{p}<0.01,{ }^{* * *} \mathrm{p}<0.001$.

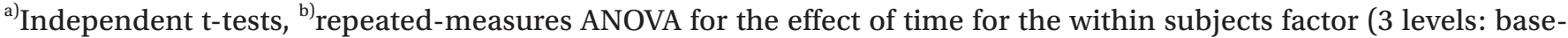
line NRS, 2 weeks later NRS, 8 weeks later NRS), ${ }^{c)}$ repeated-measures ANOVA for the effect of time for the between subjects factor (non-hyperlipidemia and hyperlipidemia).

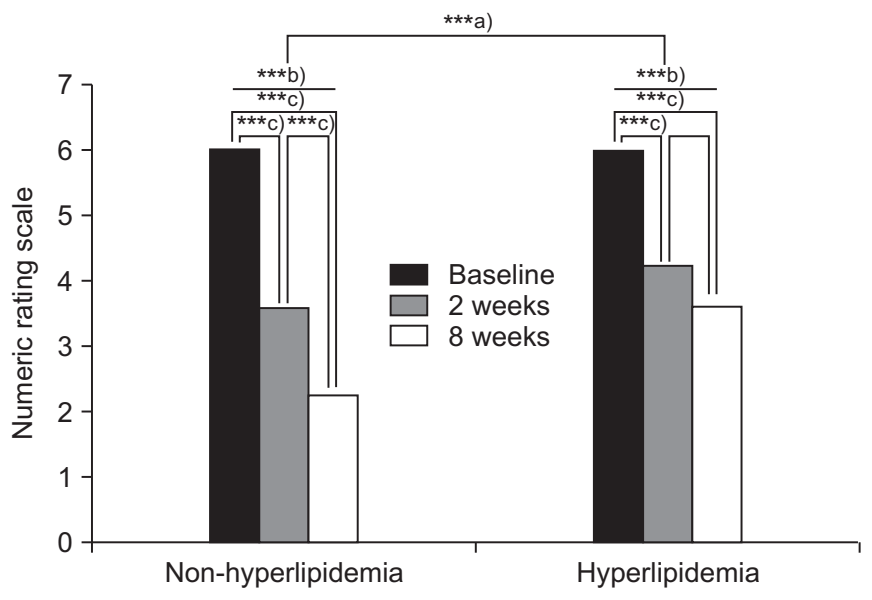

Fig. 1. Change of numeric rating scale (NRS) from the baseline to 8 weeks after, in both the groups. ${ }^{*} \mathrm{p}<0.05$, ${ }^{* *} \mathrm{p}<0.01,{ }^{* * *} \mathrm{p}<0.001,{ }^{\text {a) }}$ repeated-measures ANOVA for the effect of time for the between subjects factor (non-hyperlipidemia and hyperlipidemia), ${ }^{\text {b) }}$ repeated-measures ANOVA for the effect of time for the within subjects factor (3 levels: baseline NRS, 2 weeks later NRS, 8 weeks later NRS), ${ }^{\text {c) }}$ post-hoc test for comparison of NRS at each time points (baseline, 2 weeks after, 8 weeks after) in the both groups.

after treatment, NRSs were much higher in the hyperlipidemia group with statistical significance $(\mathrm{p}<0.001)$. On the repeated-measures ANOVA, NRS scores significantly decreased with time for both groups $(p<0.001)$. When analyzing the effect of time for the between subjects factor (non-hyperlipidemia and hyperlipidemia), there was significant difference in the treatment effect between the two groups $(\mathrm{p}<0.001)$, that is to say NRS was less decreased in the hyperlipidemia group. In the nonhyperlipidemia group, statistically significant differences of NRS scores were evident in the comparisons before treatment and 2 weeks after treatment $(\mathrm{p}<0.001)$, before
Table 3. Changes in passive range of motion (8 weeks after - initial)

\begin{tabular}{lccc}
\hline & $\begin{array}{c}\text { Non-hyper- } \\
\text { lipidemia } \\
\text { group } \\
(\mathbf{n}=\mathbf{3 3})\end{array}$ & $\begin{array}{c}\text { Hyper- } \\
\text { lipidemia } \\
\text { group } \\
(\mathbf{n}=\mathbf{2 9})\end{array}$ & p-value \\
\hline Flexion $\left(^{\circ}\right)$ & $16.21 \pm 10.97$ & $11.72 \pm 13.31$ & 0.471 \\
\hline Abduction $\left({ }^{\circ}\right)$ & $19.09 \pm 16.31$ & $15.51 \pm 19.60$ & 0.263 \\
\hline $\begin{array}{c}\text { External } \\
\text { rotation }\left({ }^{\circ}\right)\end{array}$ & $16.21 \pm 12.62$ & $10.51 \pm 13.51$ & 0.946 \\
\hline
\end{tabular}

Values are presented as mean \pm standard deviation.

treatment and 8 weeks after treatment $(\mathrm{p}<0.001)$, and 2 weeks and 8 weeks after treatment $(p<0.001)$, as evaluated by post-hoc test. In the hyperlipidemia group, there were statistically significant differences of NRS scores in comparison before treatment and 2 weeks after treatment $(\mathrm{p}<0.001)$, and before treatment and 8 weeks after treatment ( $p<0.001)$. Fig. 1 presents the changes of NRS of two groups from the baseline to 8 weeks later.

\section{Passive range of motion}

Table 3 shows PROMs at baseline and 8 weeks after treatment. Among 99 patients, only 62 patients had follow-up data of PROMs. Although there were no statistically significant differences, improvement of PROMs in the non-hyperlipidemia group was higher than that of the hyperlipidemia group.

\section{DISCUSSION}

The aim of the current study was to investigate the effect of hyperlipidemia on treatment of supraspinatus tendinopathy with or without tear, since the supraspi- 
natus tendon is the most commonly injured among the rotator cuff tendons. We postulated that hyperlipidemia adversely affects the improvement of pain from supraspinatus tendinopathy with or without tear. As expected, the improvement of pain from supraspinatus tendinopathy was less in the hyperlipidemia group than in the non-hyperlipidemia group. In addition, we analyzed changes in PROMs of two groups. Although improvement of PROMs in the non-hyperlipidemia group was higher than that of the hyperlipidemia group, there were no statistically significant differences. This may be because of only some patients involved in the analysis of PROMs.

Our results correspond with the study published by Beason et al. [7], which suggested that rotator cuff tendon healing properties are adversely affected by hypercholesterolemia in the rat model. Whereas that study was on an animal model, our study is the first clinical study in humans to assess the correlation between hyperlipidemia and the prognosis of rotator cuff tendinopathy.

We found that rotator cuff tears were more frequent in the hyperlipidemia than in the non-hyperlipidemia group. Although statistical analysis showed no significant difference, this finding corresponds with the results of a study by Abboud and Kim [6], in which patients with rotator cuff tears were more likely to have hypercholesterolemia when compared with those in the control group. It is possible that the prognosis was poorer in their hyperlipidemia group because more patients with rotator cuff tears were included in that group.

In previous investigations, an association has been reported between diabetes mellitus and rotator cuff tears. Abate et al. [12] observed a higher incidence of full-thickness rotator cuff tears in diabetic patients and Clement et al. [13] reported poorer outcomes in diabetic patients after arthroscopic repair. In our study, the prevalence of diabetes mellitus was higher in the hyperlipidemia group than in the non-hyperlipidemia group. This may have affected the result of poorer outcomes in the hyperlipidemia group. However, when comparing data with regard to the presence or absence of diabetes mellitus in the hyperlipidemia group, no significant difference of treatment effect was seen $(p=0.82)$. Furthermore, because the other baseline characteristics which may be associated with supraspinatus tendinopathy and tear were not significantly different between the two groups, these factors might not affect the treatment of supraspinatus tendinopathy with or without tear.

The mechanism of how hyperlipidemia affects healing of supraspinatus tendons remains unclear. Previous research by Beason et al. [8] demonstrated several possibilities. In one study, hypercholesterolemia was associated with increases in supraspinatus tendon stiffness and elastic modulus. Specifically, hypercholesterolemic mice, rats, and monkeys showed a significant increase in stiffness compared with controls, and elastic modulus was significantly increased in hypercholesterolemic mice and monkeys. The authors suggested that these increased properties may be inherent to the effect of hypercholesterolemia on the supraspinatus tendon rather than the result of an effect of length of time exposed to the cumulative effects of high plasma cholesterol levels. In another study, reduced elastic modulus in the mouse patellar tendon appeared to be due to lifelong exposure to high cholesterol [14].

Conversely, several studies have been conducted on the correlation between hyperlipidemia and Achilles tendon rupture. Kuriyama et al. [15] reported that patients with cerebrotendinous xanthomatosis complain more often of Achilles tendon xanthomas, which predispose to Achilles tendon rupture. Klemp et al. [16] reported that Achilles xanthomas and Achilles tendinitis were more frequent with familial hypercholesterolemia. The deposition of cholesterol byproducts, such as xanthomas, may change the mechanical properties of tendons and increase the risk of their rupture [17]. As in Achilles tendon rupture, accumulation of cholesterol byproducts in rotator cuff tendons may delay the healing of rotator cuff tendinopathy. Furthermore, hyperlipidemia is related to atherosclerosis, disturbs the blood flow, and adversely affects the nourishment of tissue. This may play a role in the healing of injured tendon [18].

From the present study, we found that improvement of pain from supraspinatus tendinopathy was less in the patients with hyperlipidemia than those without hyperlipidemia. Therefore, when supraspinatus tendinopathy is suspected, an early evaluation for hyperlipidemia may prove to be helpful. Furthermore, considering that patients with elevated serum cholesterol levels may have a reduced ability to heal from rotator cuff tendon injuries, they would benefit from a warning to avoid activities that could delay tendon healing. In a previous study, musculoskeletal manifestations from hypercholesterol- 
emia, including Achilles tendinitis, improved or resolved completely in patients after they received lipid lowering treatment [16]. Other studies proposed the use of dietary supplements, including omega-3 fatty acids and antioxidants, for potential benefits in the management of tendinopathy $[19,20]$. Thus several modalities, including lipidlowering medications, can be applied for improving lipid levels in patients with rotator cuff disease and hyperlipidemia.

Several limitations to our study exist because the data were retrospectively extracted from medical records. First, we could not control some factors affecting the healing of supraspinatus tendinopathy, such as tear size, and treatment methods. Especially, treatment methods could not be applied equally for all patients in this study. A previous prospective study applied an identical treatment protocol-triamcinolone injection, same frequency of physical therapy-for all included patients to identify the effect of corticosteroid injection in rotator cuff tears [21]. If our study was performed prospectively like the previous study, this limitation might be overcome. Second, the sample of patients included in this study does not represent the entire population of patients with supraspinatus tendinopathy because we excluded the patients who did not come for follow-up visits or laboratory studies. Third, other assessment systems capable of explaining pain or functional limitation from supraspinatus tendinopathy were not analyzed in this study. Finally, we could not definitely exclude the secondary frozen shoulder combined with supraspinatus tendinopathy. Therefore, the improvement of PROMs in this study may be due to not only recovery of supraspinatus tendinopathy, but also that of secondary frozen shoulder. Well-designed prospective studies are required to investigate interactions between hyperlipidemia and treatment effect in rotator cuff tendinopathy to overcome these limitations. Furthermore, it would be interesting to study whether medications for the management of hyperlipidemia would help in the treatment of rotator cuff tendinopathy.

In this retrospective study, we found that hyperlipidemia may be a factor adversely affecting the treatment of supraspinatus tendinopathy with or without tear. Future prospective studies are needed to reveal the influence of hyperlipidemia on treatment of supraspinatus tendinopathy or tear.

\section{CONFLICT OF INTEREST}

No potential conflict of interest relevant to this article was reported.

\section{REFERENCES}

1. Seitz AL, McClure PW, Finucane S, Boardman ND 3rd, Michener LA. Mechanisms of rotator cuff tendinopathy: intrinsic, extrinsic, or both? Clin Biomech (Bristol, Avon) 2011;26:1-12.

2. Nho SJ, Yadav H, Shindle MK, Macgillivray JD. Rotator cuff degeneration: etiology and pathogenesis. Am J Sports Med 2008;36:987-93.

3. Via AG, De Cupis M, Spoliti M, Oliva F. Clinical and biological aspects of rotator cuff tears. Muscles Ligaments Tendons J 2013;3:70-9.

4. Titchener AG, White JJ, Hinchliffe SR, Tambe AA, Hubbard RB, Clark DI. Comorbidities in rotator cuff disease: a case-control study. J Shoulder Elbow Surg 2014;23:1282-8.

5. Mall NA, Tanaka MJ, Choi LS, Paletta GA Jr. Factors affecting rotator cuff healing. J Bone Joint Surg Am 2014;96:778-88.

6. Abboud JA, Kim JS. The effect of hypercholesterolemia on rotator cuff disease. Clin Orthop Relat Res 2010;468:1493-7.

7. Beason DP, Tucker JJ, Lee CS, Edelstein L, Abboud JA, Soslowsky LJ. Rat rotator cuff tendon-to-bone healing properties are adversely affected by hypercholesterolemia. J Shoulder Elbow Surg 2014;23:867-72.

8. Beason DP, Hsu JE, Marshall SM, McDaniel AL, Temel RE, Abboud JA, et al. Hypercholesterolemia increases supraspinatus tendon stiffness and elastic modulus across multiple species. J Shoulder Elbow Surg 2013;22:681-6.

9. Bretzke CA, Crass JR, Craig EV, Feinberg SB. Ultrasonography of the rotator cuff: normal and pathologic anatomy. Invest Radiol 1985;20:311-5.

10. Jellinger PS, Smith DA, Mehta AE, Ganda O, Handelsman Y, Rodbard HW, et al. American association of clinical endocrinologists' guidelines for management of dyslipidemia and prevention of atherosclerosis. Endocr Pract 2012;18 Suppl 1:1-78.

11. Hjermstad MJ, Fayers PM, Haugen DF, Caraceni A, Hanks GW, Loge JH, et al. Studies comparing numeri- 
cal rating scales, verbal rating scales, and visual analogue scales for assessment of pain intensity in adults: a systematic literature review. J Pain Symptom Manage 2011;41:1073-93.

12. Abate M, Schiavone C, Salini V. Sonographic evaluation of the shoulder in asymptomatic elderly subjects with diabetes. BMC Musculoskelet Disord 2010; 11:278.

13. Clement ND, Hallett A, MacDonald D, Howie C, McBirnie J. Does diabetes affect outcome after arthroscopic repair of the rotator cuff? J Bone Joint Surg Br 2010;92:1112-7.

14. Beason DP, Abboud JA, Kuntz AF, Bassora R, Soslowsky LJ. Cumulative effects of hypercholesterolemia on tendon biomechanics in a mouse model. J Orthop Res 2011;29:380-3.

15. Kuriyama M, Fujiyama J, Yoshidome H, Takenaga S, Matsumuro K, Kasama T, et al. Cerebrotendinous xanthomatosis: clinical and biochemical evaluation of eight patients and review of the literature. J Neurol Sci 1991;102:225-32.

16. Klemp P, Halland AM, Majoos FL, Steyn K. Musculoskeletal manifestations in hyperlipidaemia: a con- trolled study. Ann Rheum Dis 1993;52:44-8.

17. von Bahr S, Movin T, Papadogiannakis N, Pikuleva I, Ronnow P, Diczfalusy U, et al. Mechanism of accumulation of cholesterol and cholestanol in tendons and the role of sterol 27-hydroxylase (CYP27A1). Arterioscler Thromb Vasc Biol 2002;22:1129-35.

18. Ozgurtas T, Yildiz C, Serdar M, Atesalp S, Kutluay T. Is high concentration of serum lipids a risk factor for Achilles tendon rupture? Clin Chim Acta 2003;331:258.

19. Lewis JS, Sandford FM. Rotator cuff tendinopathy: is there a role for polyunsaturated fatty acids and antioxidants? J Hand Ther 2009;22:49-55.

20. Mavrogenis S, Johannessen E, Jensen P, Sindberg C. The effect of essential fatty acids and antioxidants combined with physiotherapy treatment in recreational athletes with chronic tendon disorders: a randomised, double-blind, placebo-controlled study. Phys Ther Sport 2004;5:194-9.

21. Gialanella B, Prometti P. Effects of corticosteroids injection in rotator cuff tears. Pain Med 2011;12:155965. 